\title{
Some aspects of finite element modelling of ultrasonically aided micro-EDM of CoCr alloys
}

\author{
Daniel Ghiculescu*, Niculae Marinescu and Claudiu Pirnau \\ "Politehnica" University of Bucharest, Manufacturing Engineering Department, 313 Splaiul \\ Independentei, Bucharest, Romania
}

\begin{abstract}
The paper deals with finite element modelling of micromachining $\mathrm{CoCr}$ alloys by ultrasonically aided electrical discharge machining. This hybrid machining process has two components: a thermal one due to EDM, and a mechanical one to ultrasonic assistance. Both components were modelled using Thermal and Structural Mechanics time dependent modules of Comsol Multiphysics. The results were compared with the experimental data obtained in our laboratories, proving a good agreement and offering some solutions for machining optimization.
\end{abstract}

\section{Introduction}

The mechanical properties of CoCr alloys, extremely wear resistant and also at corrosion, and biocompatibility as well, recommend their use in medical applications, injection molding and engine components $[1,2]$. The stellite superalloys have similar using areas but also at cutting tools, components for guns etc. The $\mathrm{C}$ content and existence of carbides play an important part in their functional behavior $[3,4]$. Conventional casting of $\mathrm{CoCr}$ alloys determines existence of metallic carbides, affecting later machining, in comparison with other nonconventional techniques like selective laser melting (SLM) or Electron Beam Melting (EBM) that assures a homogenous structure [1,2].

All increased properties of $\mathrm{CoCr}$ alloys determine high difficulties at conventional machining of micro-surfaces [5]. Ultrasonically aided micro-electrical discharge machining $(\mu \mathrm{EDM}+\mathrm{US})$ is an appropriate response in terms of machining rate, precision and surface quality, supported by relative low costs [6]. This hybrid process was applied for other advanced materials difficult to machine by cutting technologies [7, 8].

At micro-EDM+US application, $\mathrm{CoCr}$ alloys thermo-physical characteristics as well as mechanical ones become critical, corresponding to their two components of this hybrid machining: thermal one due to EDM, and ultrasonically induced cavitation owed to ultrasonic assistance. In this respect, values of properties needed for modelling of EDM part are: density $8290 \mathrm{~kg} / \mathrm{m}^{3}$ for usual alloys, or much higher $\left(9120-9240 \mathrm{~kg} / \mathrm{m}^{3}\right)$ at additional alloying elements, and $8690 \mathrm{~kg} / \mathrm{m}^{3}$ for stellite, thermal conductivity $33 \mathrm{~W} / \mathrm{m}^{\circ} \mathrm{K}$ at $1000{ }^{\circ} \mathrm{C}$, which is lower than at usual steels, heat capacity $420-460 \mathrm{~J} / \mathrm{kg}{ }^{\circ} \mathrm{K}$, which is slight lower than at usual steels, melting range $1380-1430^{\circ} \mathrm{C}[5,10,11]$, and boiling temperature around $3000{ }^{\circ} \mathrm{C}$, taking into account the boiling points of alloy main components $(\mathrm{Co}, \mathrm{Cr}, \mathrm{Mo})$.

${ }^{*}$ Corresponding author: daniel.ghiculescu@upb.ro 
About mechanical characteristics of $\mathrm{CoCr}$ alloys, necessary for ultrasonic material removal modelling, modulus of elasticity varies between $200-220 \mathrm{GPa}$, much higher than other alloys used in medical applications, based on $\mathrm{Ti}$ and $\mathrm{Au}$, and ultimate tensile strength is $1300 \mathrm{MPa}[1,2,9]$. Stellite alloys have higher elasticity modulus at $230 \mathrm{GPa}$, and ultimate tensile strength at $1195 \mathrm{MPa}[3,4]$, in comparison with CoCr Arcam alloys with $960 \mathrm{MPa}$ [2]. The fatigue limit is $610 \mathrm{MPa}$ at rotating beam fatigue test [2]. Other values of fatigue limits of the alloys are: at bending, this is estimated in the region of $480 \mathrm{MPa}$ [9]; for stents $\mathrm{CoCr}$ alloys, it is predicted much lower, at $207 \mathrm{MPa}$ [10].

\section{Experimental data}

An experimental setup for preliminary studying the micro-EDM+US process on CoCr alloy was created, presented in fig. 1. This comprises basically an ultrasonic chain, integrating the tool its end, connected to an ultrasonic generator of $20 \mathrm{kHz}$, producing tool amplitude of around $2 \mu \mathrm{m}$ during EDM process. CoCr alloy rectangular samples of $30 \times 50 \times 1 \mathrm{~mm}$ were machined comparatively on Romanian ELER 01 machine with US vibrations of tool (EDM+US) and without US (classic EDM).

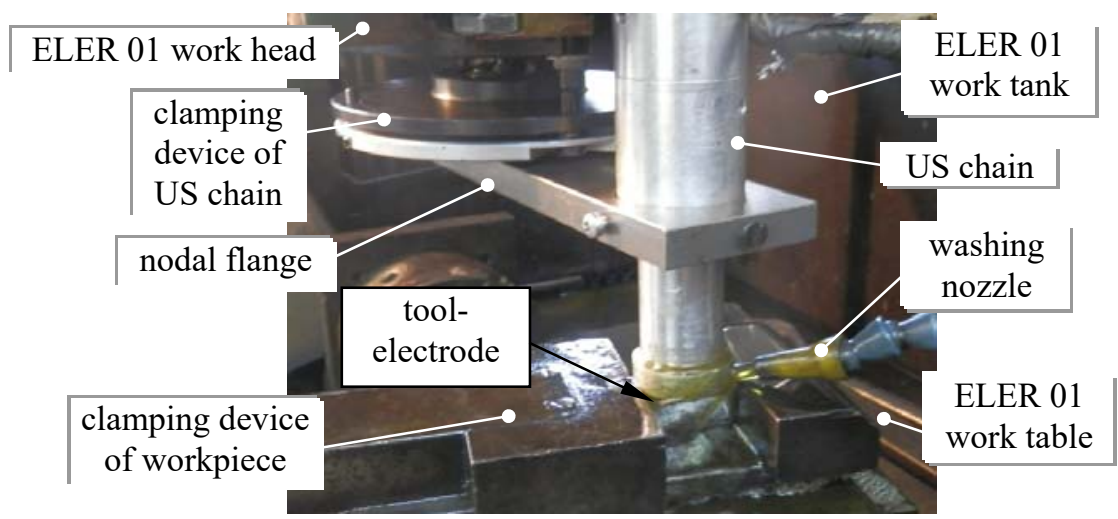

Fig. 1. Technological system elements for EDM+US micromachining of $\mathrm{CoCr}$ alloy samples.

Both commanded and relaxation pulses were used provided by a special generator for finishing and micro-machining. The working parameters at commanded pulses were: current step, $I=3 \mathrm{~A}$, positive (tool) polarity; pulse time, $t_{i}=48 \mu \mathrm{s}$, pause time, $t_{0}=24 \mu \mathrm{s}$; $t_{i}=8 \mu \mathrm{s} t_{0}=8 \mu \mathrm{s}$. At relaxation pulses, the specific working parameters were: negative polarity, capacitor step, $C=10 \mathrm{nF}$, resistance step, $R=0.74 \mathrm{k} \Omega$ (for adjusting supply current). For flushing, the lateral one was chosen with pressure, $p_{h l}=0.04 \mathrm{MPa}$. The values of consumed power on ultrasonic chain $\left(P_{c U S}\right)$ were: 90 and $120 \mathrm{~W}$.

The variation of total hydraulic pressure $\left(p_{h t}\right)$ is calculated with the following relation, and presented in fig. 2:

$$
p_{h t}=2 \pi c \rho f_{U S} z+p_{h l} \quad[\mathrm{~Pa}]
$$

where: $f_{U S}$ is ultrasonic frequency $[\mathrm{Hz}] ; z$ - elongation, $z=A \sin \omega t ; \omega=2 \pi f_{U S}\left[\mathrm{~s}^{-1}\right] A$ amplitude $[\mathrm{m}] ; c$ - sound velocity in dielectric liquid $[\mathrm{m} / \mathrm{s}] ; \rho$ - dielectric liquid density, $\rho=840 \mathrm{~kg} / \mathrm{m}^{3}\left[\mathrm{~kg} / \mathrm{m}^{3}\right] ; \quad c=(K / \rho)^{1 / 2}=1267.7 \mathrm{~m} / \mathrm{s} ; K=1.35 \times 10^{9} \mathrm{~Pa}\left(K\right.$-bulk modulus); $p_{h l}$ - local hydraulic pressure $[\mathrm{Pa}], p_{h l}=0.04 \mathrm{MPa}$. At each final of ultrasonic period $T_{U S}$, when pressure increases, cumulative microjets stage (CMS) occurs, due to the collective implosion of gas bubbles, generating shock waves. This produces pressure of $100 \mathrm{MPa}$ order of magnitude, oriented along the gap, frontal in this case - fig. 2. 


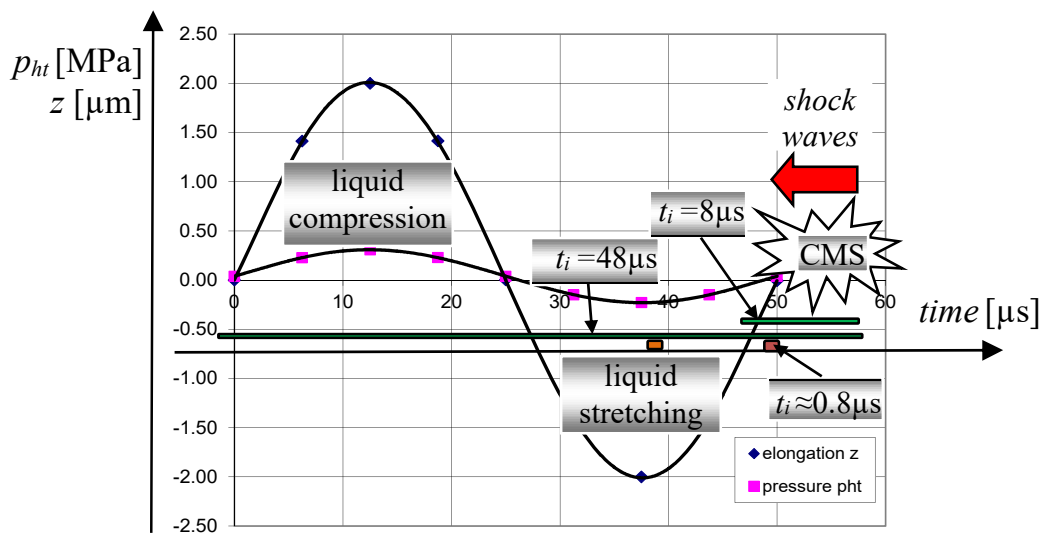

Fig. 2. Variation of tool elongation and total pressure in the gap within $T_{U S}$ during EDM+US tests.

During $T_{U S}$, commanded and relaxation pulses are delivered. At overlapping the pulses on $C M S$, it was noticed experimentally that the discharge is stopped due to high pressure generated by collective gas bubble implosion. Then the dielectric liquid is allowed to access the melted material by discharge, and therefore increased volume of material is removed.

The microtopographies provided by scanning electron microscope (SEM) QUANTA INSPECT F50, with field emission electron gun and resolution of $1 \mathrm{~nm}$, and corresponding profile of craters average dimensions, obtained by mediation of craters diameters with their depth $(\mathrm{Rz})$ given by surface roughness tester SJ411, are presented in fig. $3-8$.

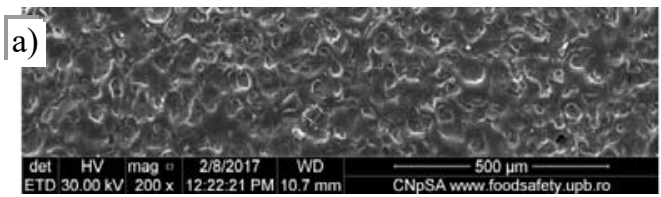

b)

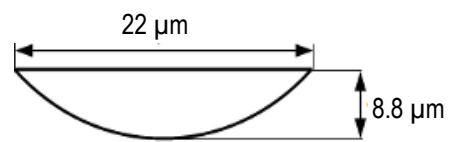

Fig. 3. Surface microgeometry at EDM with commanded pulses, $I=3 \mathrm{~A}, t_{i}=48 \mu \mathrm{s}, t_{0}=24 \mu \mathrm{s}$ :

(a) SEM view; b) craters averaged dimensions.

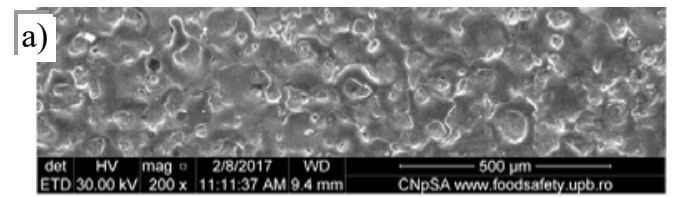

b)

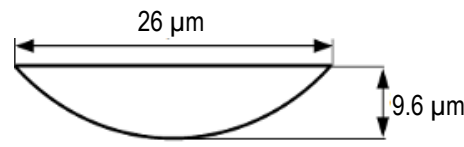

Fig. 4. Surface microgeometry at $\mu \mathrm{EDM}+\mathrm{US}$ with commanded pulses, $P_{c u S}=120 \mathrm{~W}, I=3 \mathrm{~A}, t_{i}=48 \mu \mathrm{s}$, $t_{0}=24 \mu$ s: (a) SEM view; b) craters averaged dimensions.

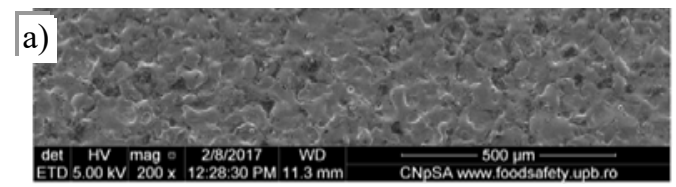

b)

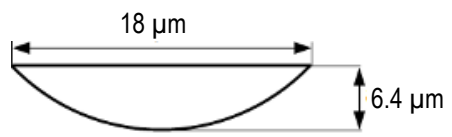

Fig. 5. Surface microgeometry at $\mu \mathrm{EDM}$ with commanded pulses, $I=3 \mathrm{~A}, t_{i}=8 \mu \mathrm{s}, t_{0}=8 \mu \mathrm{s}$ :

(a) SEM view; b) craters averaged dimensions

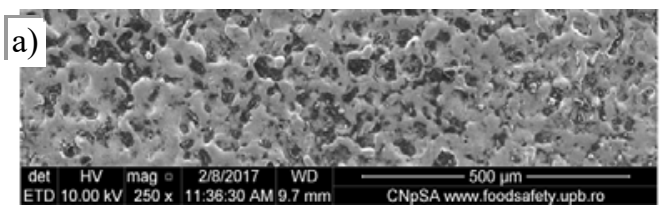

b)

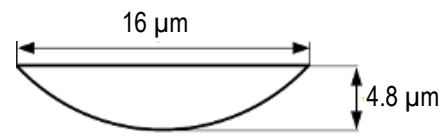

Fig. 6. Surface microgeometry at $\mu \mathrm{EDM}+\mathrm{US}$ with commanded pulses, $P_{c U S}=90 \mathrm{~W}, I=3 \mathrm{~A}, t_{i}=8 \mu \mathrm{s}$, $t_{0}=8 \mu \mathrm{s}$ : (a) SEM view; b) craters averaged dimensions. 


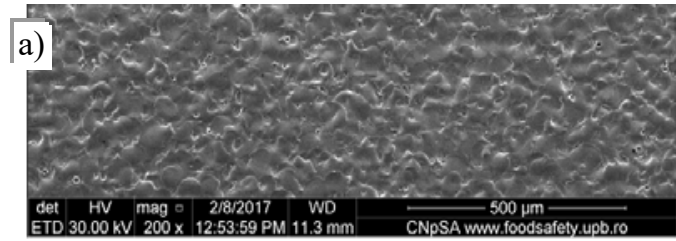

b)

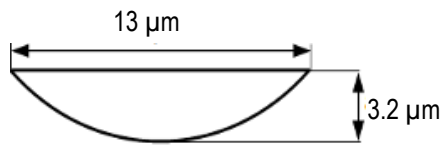

Fig. 7. Surface microgeometry at $\mu \mathrm{EDM}$ with relaxation pulses, $C=10 \mathrm{nF}, R=0.74 \mathrm{k} \Omega$ :

(a) SEM view; b) craters averaged dimensions.

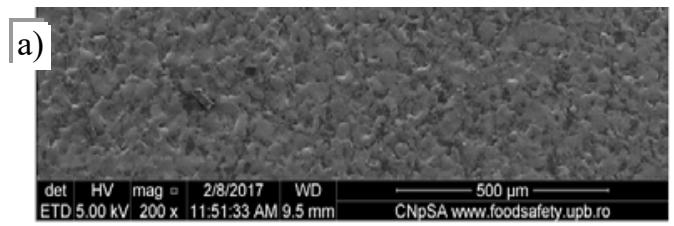

b)

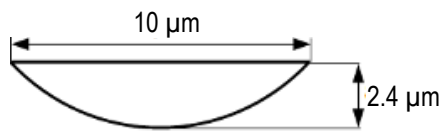

Fig. 8. Surface microgeometry at $\mu \mathrm{EDM}+\mathrm{US}$ with relaxation pulses, $P_{c U S}=90 \mathrm{~W}, C=10 \mathrm{nF}, R=0.74$ $\mathrm{k} \Omega$ : (a) SEM view; b) craters averaged dimensions.

\section{Finite element modelling of micro-EDM+US process}

The material removal process of $\mu \mathrm{EDM}+\mathrm{US}$ on $\mathrm{CoCr}$ alloys was modelled by finite element method using Comsol Multiphysics, time dependent modules of Heat Transfer in Solids and Solid Mechanics, corresponding to EDM, and ultrasonic components. Some parametric models were created for each pulse type, using the assignation shown in fig. 9.

Parameters
\begin{tabular}{|llll|}
\hline Name & Expression & Value & Description \\
\hline Ip & $10[\mathrm{~mm}]$ & $0.01 \mathrm{~m}$ & workpiece length \\
$\mathrm{hp}$ & $1[\mathrm{~mm}]$ & $0.001 \mathrm{~m}$ & workpiece height \\
$\mathrm{acr}$ & $11 \mathrm{e}-6$ & $1.1 \mathrm{E}-5$ & radius of initial craters \\
$\mathrm{bcr}$ & $4^{\star} 2.2 \mathrm{e}-6$ & $8.8 \mathrm{E}-6$ & depth of initial craters \\
$\mathrm{rms}$ & $0.1 \mathrm{e}-6$ & $1.0 \mathrm{E}-7$ & radius of resolified material \\
$\mathrm{rgb}$ & $0.1[\mathrm{~mm}]$ & $1.0 \mathrm{E}-4 \mathrm{~m}$ & radius of gas bubble \\
$\mathrm{ti}$ & $10 \mathrm{e}-6$ & $1.0 \mathrm{E}-5$ & discharge duration \\
modulE & $220[\mathrm{GPa}]$ & $2.2 \mathrm{E} 11 \mathrm{~Pa}$ & Young's modulus \\
\hline $\mathrm{PR}$ & 0.29 & 0.29 & Poisson ratio \\
\hline pus & $150[\mathrm{MPa}]$ & $1.5 \mathrm{E} 8 \mathrm{~Pa}$ & ultrasonic shock pressure \\
tb & $0.8 \mathrm{e}-6$ & $8.0 \mathrm{E}-7$ & bubbles implosion time \\
\hline
\end{tabular}

Fig. 9. Example of assigned parameters for material removal process of $\mu \mathrm{EDM}+\mathrm{US}$ on $\mathrm{CoCr}$ alloy.

The Van Dijck and Snoeys's overheating model was considered for EDM component modelling. In this case, boiling is the main mean to remove overheated material after pulse end when pressure drops suddenly; the gas bubble formed around plasma channel lasts very long after the end of pulse, so the material melted by discharge is already solidified [6].

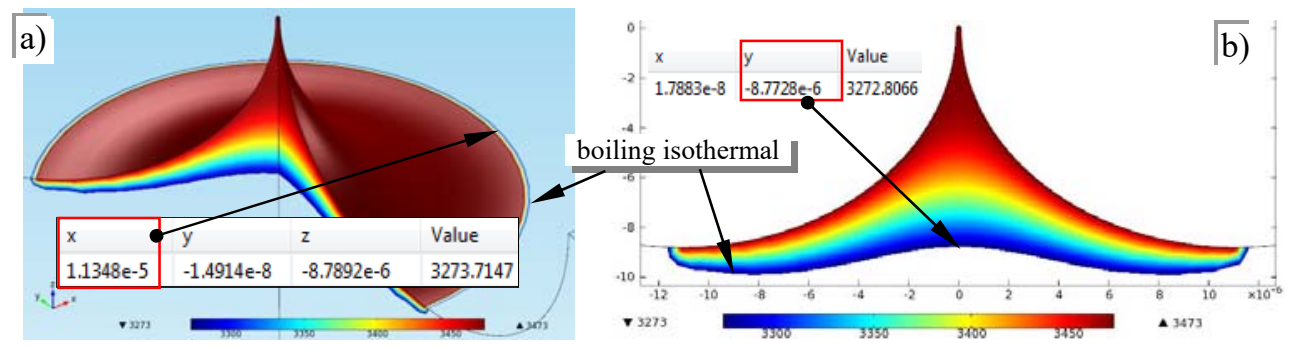

Fig. 10. Removed material, boiling isothermal [K] disposal at $\mu \mathrm{EDM}$ after $t_{i}=48 \mu \mathrm{s}$ : (a) 3D; b) section view. 
Constant $3473 \mathrm{~K}$ temperature on EDM spot, thermal isolation on area covered by gas bubble, and convection cooling on workpiece periphery in contact with dielectric liquid with $313 \mathrm{~K}$ were setup as boundary conditions. The temperature distribution obtained around EDM spot with $t_{i}=48 \mu \mathrm{s}$ and $t_{0}=24 \mu \mathrm{s}$ is presented in fig. 10 , indicating close value to experimental ones (see fig. 3.b), validating the model.

At ultrasonic assistance, due to relative long pulse time $t_{i}=48 \mu \mathrm{s}$, comparable with $T_{U S}$, it is very high probability to its overlapping on $C M S$ [14]. So, melting becomes the main mechanism of material removal since the gas bubble around plasma channel collapses at $C M S$, and dielectric liquid accesses the melted material. The temperature distribution obtained in this case is presented in fig. 11.a:

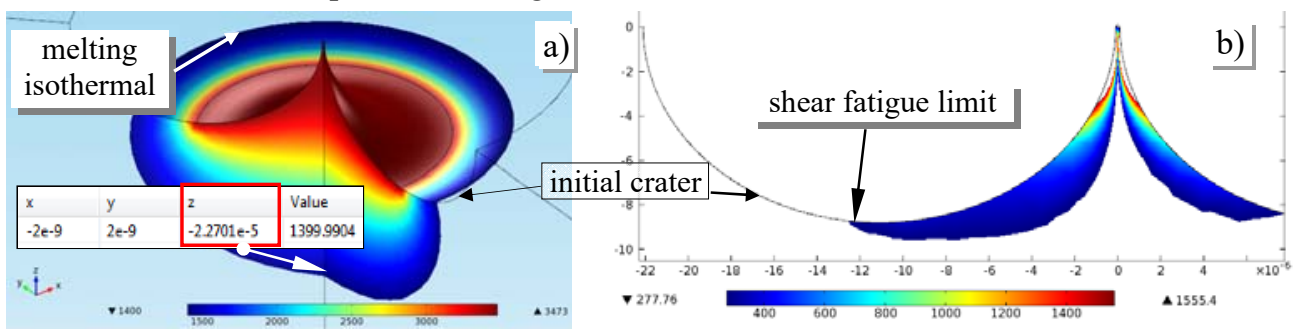

Fig. 11. Removed material at $\mu \mathrm{EDM}+\mathrm{US}$ : a) melting isothermal $[\mathrm{K}]$ disposal after $t_{i}=10 \mu \mathrm{s}$ from pulse start, at $C M S$; b) Von Mises stress [MPa] at $150 \mathrm{MPa}$ ultrasonic pressure shock wave.

During EDM removal, another mechanism is produced due to ultrasonic cavitation. Crater margins are removed by shear of shock waves oriented parallel to machined surface. In order to underline the volume of material removed by hydraulic mechanical effect of ultrasonic cavitation, the fatigue limit at shear pulsing cycle $\left(\tau_{0}\right)$ was calculated [11]:

$$
\tau_{0}=1.12\left(40+0.16 \sigma_{r}\right), \quad[\mathrm{MPa}]
$$

where: $\sigma_{r}$ is the usual ultimate tensile strength; in this case, $\sigma_{r}=1300 \mathrm{MPa}$, and the parameter of interest is $\tau_{o}=277.76 \mathrm{MPa}$. In fig. 11.b, it can be observed a massive removal also from the bottom of crater, which determines the roughness increase, i.e. the depth of crater. This is confirmed by experimental data (see fig. 4.b), explained by relative high power, $P_{c U S}=$ $120 \mathrm{~W}$ used on ultrasonic chain. Both removal mechanism determined increase of craters dimensions in comparison with pure micro-EDM, experimentally emphasized by fig. 4 .

At $\mu \mathrm{EDM}+\mathrm{US}$ with $t_{i}=8 \mu \mathrm{s}, t_{0}=8 \mu \mathrm{s}$, the overlapping probability of pulse time on CMS, decreases very much, under $20 \%$ [6]. So, classic thermal removal like in classic $\mu$ EDM occurs dominantly, and the temperature distribution after pulse end is presented in fig. 12:

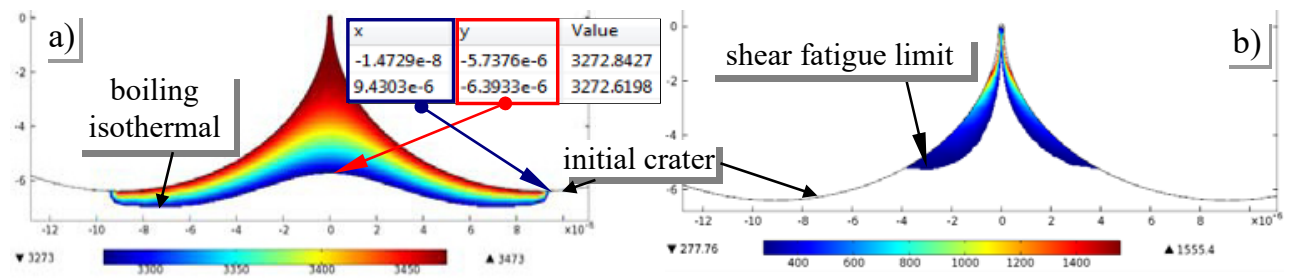

Fig. 12. Material removed at $\mu \mathrm{EDM}+\mathrm{US}, t_{i}=8 \mu$ s commanded pulse: a) boiling isothermal $[\mathrm{K}]$ disposal; b) Von Mises stress [MPa] at pulse cycling at $110 \mathrm{MPa}$ ultrasonic pressure shock wave.

The boiling isothermal position is in agreement with experimental data from fig. 5, validating the model. Over this thermal removal mechanism is superimposed, the ultrasonic removal due to shock wave produced by $C M S$ parallel to machined surface [6]. In fig. 12.b, distribution of Von Mises stress is presented, higher than shear fatigue limit, which shows 
the material that is removed. The decrease of craters depth is produced, and implicitly the surface roughness, confirming the experimental data from fig. 6.b, at lower $P_{c U S}=90 \mathrm{~W}$.

At $\mu \mathrm{EDM}+\mathrm{US}$ with relaxation pulses, the nature of material removal mechanism is very similar with the previous working mode. The ultra-short discharge duration of relaxation pulses makes a very low probability to overlap them on $C M S$. So, classic thermal removal is dominant, as presented in fig. 13.a, associated with ultrasonic shear of craters margins, producing lowering of craters dimensions, confirmed experimentally by data from fig. 8 .

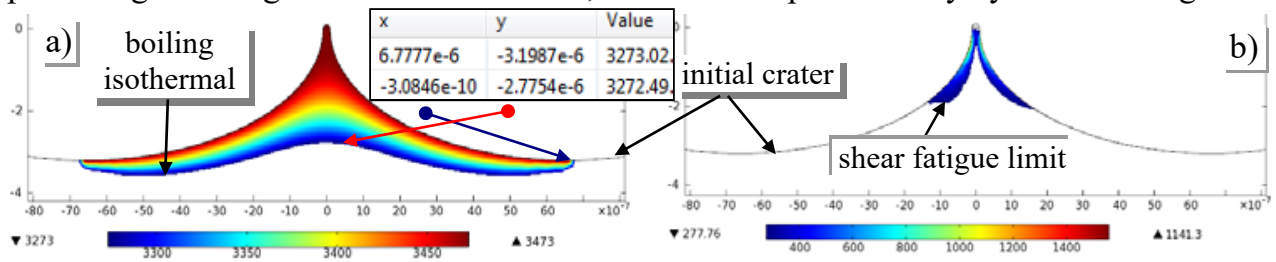

Fig. 13. Material removed at $\mu E D M+U S$, relaxation pulse: a) boiling isothermal $[K]$ disposal after pulse end; b) Von Mises stress [MPa] at pulse cycling at $110 \mathrm{MPa}$ ultrasonic pressure shock wave.

\section{Conclusions}

The FEM study of $\mu$ EDM+US on CoCr alloy, not systematically treated in the state of the art yet, emphasized two types of material removal mechanism, confirmed by experimental data. At longer pulse time, comparable with ultrasonic period, the thermal mechanism of removal is dominant, and position of melting isothermal shows a spectacular increase of machining rate. At shorter pulse time, especially at relaxation pulses using, the ultrasonic hydraulic removal becomes dominant by removing the craters margins - the debris are not spherical like at melting and resolidification. Finding an optimum value of consumed power on ultrasonic chain, correlated with $\mathrm{CoCr}$ alloy fatigue limit, is of utmost importance in order to decrease the surface roughness in comparison with pure $\mu$ EDM.

The paper was achieved in Joint Applied Research Project supported by MEN-UEFISCDI, project no. PN-II-PT-PCCA-2013-4-0236, Contract no. 222/2014. The SEM analyzes/images obtained on the samples were possible due to EU-funding project POSCCE-A2-O2.2.1-2013-1, SMIS-code 48652.

\section{References}

1. Y. S. Al Jabbari, J. Adv. Prosthodont. 6, 138-145 (2014)

2. $* * *$ Arcam AB, ASTM F75 CoCr Alloy, 3 (2010)

3. S. Kapoor, R. Liu, X. J. Wu, M. X. Yao, Int. J. Ch. M. Nucl. Mat. Met. Eng. 6, 592$601(2012)$

4. $* * *$ Hardfacing Alloys 22, kennametal.com/stellite (2016)

5. S. Baron, E. Ahearne, P. Connolly, S. Keaveney, G. Byrne, Proc. Mach. Tool Tech. Res. Found., 1-7 (2015)

6. D. Ghiculescu, N.I. Marinescu, O. Alupei, Applied Mechanics and Materials, 834, 126-131 (2016)

7. M. Iwai, S. Ninomiya, K.Suzuki, Procedia CIRP, 6, 146-150 (2013)

8. Z. Qinjian, Z. Luming, L. Jianyong, et al., Procedia CIRP, 6, 589-593 (2013)

9. $* * *$ Cobalt Chrome Alloy Co28Cr6Mo, Material specifications, 3T RPD Ltd. UK (2012)

10. R. V. Marreya, R. Burgermeistera, R. B. Grishabera, R.O. Ritchie, Biomaterials 27, 1988-2000 (2006)

11. V. Drobota, Resistance of Materials, (Didactic and Pedagogical, Bucharest, 1982) 